\title{
SIEVE METHODS FOR ODD PERFECT NUMBERS
}

\author{
S. ADAM FLETCHER, PACE P. NIELSEN, AND PASCAL OCHEM
}

\begin{abstract}
Using a new factor chain argument, we show that 5 does not divide an odd perfect number indivisible by a sixth power. Applying sieve techniques, we also find an upper bound on the smallest prime divisor. Putting this together we prove that an odd perfect number must be divisible by the sixth power of a prime or its smallest prime factor lies in the range $10^{8}<p<10^{1000}$. These results are generalized to much broader situations.
\end{abstract}

\section{INTRODUCTION}

A positive integer of the form $2^{n}-1$ is called a Mersenne number. These numbers can only be prime when $n$ is prime. As it currently stands, there are forty-seven known Mersenne primes. The largest is $2^{43112609}-1$, which is also currently the largest explicitly known prime, and the first such found with more than ten-million digits. As observed by Euclid, a number of the form $N=2^{p-1}\left(2^{p}-1\right)$, where $2^{p}-1$ is a Mersenne prime, possesses the property that $\sigma(N)=2 N$, and Euler proved the converse in the case when $N$ is even. A positive integer $N$ satisfying $\sigma(N)=2 N$ is called a perfect number. There are two questions, open since antiquity, concerning perfect numbers.

Question 1. Are there infinitely many even perfect numbers?

Equivalently, one can ask if there are infinitely many Mersenne primes. Due to heuristic arguments, and the fact that prime-hunters keep finding more of them at about the right times, the widely held belief is that the answer to this question is yes. However, little concrete progress has been made in formally answering the question. A positive answer would give an answer to other difficult open questions, such as whether or not the quadratic polynomial $2 x^{2}-1$ is prime infinitely often. Those wishing to look for Mersenne primes can participate in GIMPS (the Great Internet Mersenne Prime Search) by downloading a free program that runs on spare computer cycles from http://www.mersenne.org.

Question 2. Are there any odd perfect numbers?

The popular opinion is that the answer is no. Further, there are a number of known limiting conditions. We give an updated version of the list provided in [24]. Write $N=\prod_{i=1}^{k} p_{i}^{a_{i}}$ where each $p_{i}$ is prime, $p_{1}<p_{2}<\ldots<p_{k}$, and $k=\omega(N)$ is the number of distinct prime factors. Then:

- Eulerian Form: We have $N=\pi^{\alpha} m^{2}$ for some integers $\pi, \alpha, m \in \mathbb{Z}_{>0}, \pi \nmid m$, with $\pi \equiv \alpha \equiv 1(\bmod 4)$ and $\pi$ prime. The prime $\pi$ is called the special prime of $N$.

Received by the editor April 6, 2011 and, in revised form, May 27, 2011.

2010 Mathematics Subject Classification. Primary 11A25; Secondary 11N36, 11A51, 11Y99.

Key words and phrases. Abundance, factor chains, large sieve, odd perfect number.

(C) 2012 American Mathematical Society Reverts to public domain 28 years from publication 
- Lower Bound: Brent, Cohen, and te Riele [2] using a computer search found that $N>10^{300}$. As of this writing, the third named author has completed the calculations which improve this bound to $N>10^{1500}$, and further progress is likely. William Lipp is helping coordinate this effort via his website http: //www.oddperfect.org.

- Upper Bound: Dickson [6] proved that there are finitely many odd perfect numbers with a fixed number of distinct prime factors. Pomerance [26] gave an effective bound in terms of $k$. This was improved in succession by Heath-Brown [13], Cook [5], and finally by the second author to $N<2^{4^{k}}$. Recently, Pollack [25] used these bounds to give an effective bound on the number of odd perfect numbers in terms of $k$.

- Large Factors: Goto and Ohno 9 proved that $p_{k}>10^{8}$, and Iannucci [14, 15] proved $p_{k-1}>10^{4}$ and $p_{k-2}>10^{2}$.

- Small Factors: The smallest prime factor satisfies $p_{1}<\frac{2}{3} k+2$ as proved by Grün [11. For $2 \leqslant i \leqslant 6$, Kishore [20] showed that $p_{i}<2^{2^{i-1}}(k-i+1)$, and this has been slightly improved by Cohen and Sorli [3].

- Number of Total Prime Factors: Hare [12] proved that the total number of (not necessarily distinct) prime factors of $N$ must be at least 75 . The third author is performing computations which increase this bound to 101 .

- Number of Distinct Prime Factors: The second author 24] demonstrated that $\omega(N) \geqslant 9$ and if $3 \nmid N$ then $\omega(N) \geqslant 12$. In a work in progress these bounds are increased further.

- The Exponents: Set $d=\operatorname{gcd}_{i}\left(a_{i}+1\right)$, where the $a_{i}$ run over the exponents on the non-special primes. McDaniel $[23$ ] proved that $d \not \equiv 0(\bmod 3)$. If the exponents of the non-special primes are all equal (to $d-1$ ), Yamada [30. proved that there is an effective upper bound on $N$ depending only on $d$. In this case, a number of authors have demonstrated that certain values of $d$ are unacceptable.

The focus of this paper will be on the exponents of a purported odd perfect number, the last of the bulleted points above. As above, let $d=\operatorname{gcd}_{i}\left(a_{i}+1\right)$, where the $a_{i}$ run over the exponents on the non-special primes. With modern computing power it is a simple matter to show that $d \not \equiv 0(\bmod 3)$; taking fewer than 30 cases to check. We even provide such a proof later in the paper. If we replace 3 by a larger prime, like 5, already the computation becomes much more difficult, and previously had never been accomplished. Nonetheless, there is still a straightforward algorithm to deal with this case; assuming you have a large amount of computational power at hand. However, that leaves open what happens in the case $d=\operatorname{gcd}_{i}\left(a_{i}+1\right)=1$. For example, it is known that the non-special primes cannot all have an exponent of 2 , nor can they all have exponent 4 , but it is unknown what happens in the mixed case when we allow both 2 and 4 to occur.

A new idea was developed by Yamada [29] in a preprint available at the mathematics preprint server http://arxiv.org since 2005. Using sieve methods, he proved the following:

Yamada's Theorem. Let $n, d, b_{1}, b_{2}, \ldots, b_{t}$ be positive integers, where the $b_{j}$ belong to a finite set $\mathcal{P}$. If $N=p_{1}^{a_{1}} \cdots p_{s}^{a_{s}} q_{1}^{b_{1}} \cdots q_{t}^{b_{t}}$ is the prime factorization of $N$ and $\sigma(N) / N=n / d$, then $N$ has a prime factor smaller than a constant $C$, effectively computable in terms of $n, s$, and $\mathcal{P}$. 
Yamada explicitly computed the constant $C$ in the case that $N$ is an odd perfect number (i.e. $n=2$ and $d=1$ ) where the exponents of the non-special prime factors are either 2 or 4 (so $s \leq 1$ and $\mathcal{P}=\{2,4\}$ ). The constant he obtained was $\exp \left(4.97401 \cdot 10^{10}\right)>10^{20,000,000,000}$.

In this paper we strengthen Yamada's theorem by weakening the condition "the $b_{j}$ belong to a finite set" to "there is a finite set of primes so that $b_{j}+1$ is divisible by one of them." Furthermore, we simplify the proof a great deal, and the computable constant obtained is much smaller.

Applying our theorem, we arrive at the following:

Main Theorem. Let $N$ be an odd perfect number such that if $p^{a} \| N$ and $p$ is not the special prime, then either $3 \mid(a+1)$ or $5 \mid(a+1)$. The smallest prime factor of $N$ belongs to the range $10^{8}<p<10^{1000}$.

In Sections 2 4 we develop the machinery necessary to establish an improved version of Yamada's theorem. Emphasis is given to simplifying the computations, rather than optimizing constants. Sections [5] and [6] describe how the lower bound of the main theorem is pushed to $10^{8}$. The most difficult problem, and the real heart of the paper, is dealing with the case when $p=5$. In Sections 7 [10 we finish the proof of the upper bound in the main theorem. The last few sections focus on open questions and possible future improvements.

\section{BREAK-DOWN OF THE MAIN IDEAS}

We begin with some standard notation. We define the function $\sigma_{-1}(a)=\frac{\sigma(a)}{a}=$ $\sum_{d \mid a} d^{-1}$ for positive integers $a$, and note that this function is multiplicative. Clearly, an integer $N$ is perfect if and only if $\sigma_{-1}(N)=2$. Given a prime $p$ we have $\sigma_{-1}\left(p^{a}\right)=\frac{p^{a+1}-1}{p^{a}(p-1)}$ which is increasing in $a$ and $\lim _{a \rightarrow \infty} \sigma_{-1}\left(p^{a}\right)=\frac{p}{p-1}$.

An integer $N>1$ is said to be $n / d$-perfect if $\sigma_{-1}(N)=n / d$. Reducing if necessary, we will always assume $n$ and $d$ are relatively prime positive integers. In the case $n=2$ and $d=1$, we let $\pi$ denote the special prime factor of $N$. We also let $\Phi_{m}(x)$ denote the $m$ th cyclotomic polynomial.

Hereafter, we fix an integer $s \geq 0$ and a finite set of primes $\mathcal{P}$. We will think of the set $\mathcal{P}$ as limiting the exponents of all but $s$ of the prime factors of $N$, but to make this more formal we need further notations. We set $P=\prod_{p \in \mathcal{P}} p$ and let $P_{\text {max }}$ denote the largest prime in $\mathcal{P}$. We let $N$ be an $n / d$-perfect number with a prime factorization

$$
N=\prod_{i=1}^{s} r_{i}^{a_{i}} \prod_{j=1}^{t} q_{j}^{b_{j}}
$$

where for each $1 \leq j \leq t$ there is a prime $p_{j} \in \mathcal{P}$ with $p_{j} \mid\left(b_{j}+1\right)$. In particular, we have $\Phi_{p_{j}}\left(q_{j}\right) \mid \sigma\left(q_{j}^{b_{j}}\right)$, for any such prime $p_{j} \in \mathcal{P}$. For each $p \in \mathcal{P}$ we let $Q_{p}=\{q$ : $q^{b}|| N$ and $\left.p \mid(b+1)\right\}$. Notice that prime factors of $N$ may occur in more than one of the sets $Q_{p}$, but (by design) there are at most $s$ prime factors of $N$ which belong to none of them. Without loss of generality, we also assume $r_{1}<r_{2}<\ldots<r_{s}$. We speak of the primes $q_{j}$ as the constrained primes, as their exponents are constrained to specific residue classes. The primes $r_{i}$ are unconstrained, but limited in number.

We will construct a constant, which we call $C$, that is an upper bound on the smallest prime factor of $N$. The definition of $C$ is done in three stages; we will assume that $C$ is the smallest constant satisfying the conditions given in each stage. 
For the first stage we simply assume $C \geq \max \left\{P_{\max }, n\right\}+1$. This takes care of the case that one of $n, d$, or $P$ shares a prime factor in common with $N$. Hereafter, we will assume that $N$ does not share such a factor. Noting that $\sigma(N)$ is an integer, we derive from the equation $\sigma(N)=N n / d$ that $d \mid N$, and hence we may now assume $d=1$.

The second stage is also straightforward, and yields a large improvement over 29]. From the equation $\sigma_{-1}(N)=n$ we have

$$
\begin{aligned}
n=\prod_{i=1}^{s} \sigma_{-1}\left(r_{i}^{a_{i}}\right) \prod_{j} \sigma_{-1}\left(q_{j}^{b_{j}}\right) & \leq \prod_{i=1}^{s} \frac{r_{1}+i-1}{r_{1}+i-2} \prod_{p \in \mathcal{P}} \prod_{q \in Q_{p}} \frac{q}{q-1} \\
& =\frac{r_{1}+s-1}{r_{1}-1} \prod_{p \in \mathcal{P}} \prod_{q \in Q_{p}} \frac{q}{q-1} .
\end{aligned}
$$

We wish to turn this inequality into a non-trivial lower-bound on the quantity $\prod_{p \in \mathcal{P}} \prod_{q \in Q_{p}} \frac{q}{q-1}$. This occurs only when $n>\frac{r_{1}+s-1}{r_{1}-1}$. Equivalently, we need $r_{1}>\frac{s+n-1}{n-1}$. For ease later, we define the following constants: $C_{0}=\frac{s+n-1}{n-1}$ and

$$
C_{0}^{\prime}=\frac{n\left(r_{1}-1\right)}{s+r_{1}-1} \leq \prod_{p \in \mathcal{P}} \prod_{q \in Q_{p}} \frac{q}{q-1} .
$$

Our second assumption is $C \geq C_{0}+1$. The observant reader will note that the constant $C_{0}^{\prime}$ depends on more than just $n, s$, and $\mathcal{P}$; namely the prime $r_{1}$. However, note that with the assumption $C \geq C_{0}+1$ in place we lose no generality by assuming $r_{1}>C_{0}$. Thus, we also have the inequality $C_{0}^{\prime}>1$, and $C_{0}^{\prime}$ is bounded away from 1 by a positive quantity only depending on $n, s$, and $\mathcal{P}$.

The third stage, which is explained fully in the next two sections, relies on the following idea: We wish to count the number of prime factors of $N$ which are congruent to $1(\bmod P)$. To this end, fix one of the constrained primes $q \in Q_{p}$. If $q \equiv 1(\bmod P)$, then $p\left|\Phi_{p}(q)\right| \sigma\left(q^{b}\right) \mid \sigma(N)=N n$. From our first assumption on $C$, we know $p \nmid N$, hence $p \mid n$. Thus there are at most $v_{p}(n)$ primes $q \in Q_{p}$ with $q \equiv$ $1(\bmod P)$, where $v_{p}$ denotes the $p$-adic valuation. In particular, there are at most $C_{1}=s+\sum_{p \in \mathcal{P}} v_{p}(n)$ distinct prime factors of $N$ which are $\equiv 1(\bmod P)$. We will use the large sieve to show that this constraint implies that $\prod_{p \in \mathcal{P}} \prod_{q \in Q_{p}} q /(q-1)$ converges quickly. If this double product involves only sufficiently large primes, then we can guarantee it is smaller than $C_{0}^{\prime}$, which yields a contradiction.

\section{The LARGE SIEVE}

In this section we fix a prime $p_{0} \in \mathcal{P}$ and we let $p$ represent an arbitrary prime. To use the large sieve we first need to construct appropriate sieving sets. For $p \equiv 1(\bmod P)$ we set

$$
\Omega_{p}=\{0(\bmod p)\} \cup\left\{a(\bmod p): a \not \equiv 1(\bmod p), a^{p_{0}} \equiv 1(\bmod p)\right\},
$$

and for all other primes we set $\Omega_{p}=\{0(\bmod p)\}$. We wish to estimate the size of the set $\mathcal{S}=\left\{n \leq x: n \notin \Omega_{p}\right.$ for all $\left.p<u\right\}$, where $x, u \in \mathbb{R}_{>0}$.

To see why we want to bound the size of $\mathcal{S}$ consider the following argument. Assume $q \in Q_{p_{0}}, q \not \equiv 1(\bmod p)$, and $q^{p_{0}} \equiv 1(\bmod p)$. From the definition of $Q_{p_{0}}$, this implies that $p \mid N$. If we further assume that $C_{1}=0$, then there are no primes $p \mid N$ with $p \equiv 1(\bmod P)$. Thus, under the assumptions above, $q \notin \Omega_{p}$ unless $q=p$. In particular, we have the upper-bound $\left|\left\{q \in Q_{p_{0}}: q<x\right\}\right| \leq|\mathcal{S}|+u$. Since $C_{1}$ is 
not necessarily 0 a small corrective factor needs to be introduced, which we do at the end of this section.

If $p \equiv 1(\bmod P)$ we set $\kappa(p)=p_{0}$. Otherwise we set $\kappa(p)=1$. We extend $\kappa$ multiplicatively, and define it to be zero on non-square-free arguments. Note that $\kappa(p)=\left|\Omega_{p}\right|$. We now describe how to give an effective bound on $|S|$.

Proposition 1 (The Large Sieve [16, Theorem 7.14]). We have

$$
|\mathcal{S}| \leq \frac{x+u^{2}}{G(u)}
$$

where $G(u)=\sum_{n \leq u} \mu^{2}(n) \prod_{p \mid n}\left(\frac{\kappa(p)}{p-\kappa(p)}\right)$.

Note that we are in the situation where "small sieves" would apply, and this is the avenue Yamada explored. However, to avoid complications with error-term estimates we prefer the large sieve. In the sieve we take $u=\sqrt{x}$, and thus we need to find a lower-bound for $G(\sqrt{x})$. Set $V(P(w))=\prod_{p<w}\left(1-\frac{\kappa(p)}{p}\right)$. One can approximate $G(u)$ from above and below by suitable multiples of $V(P(w))^{-1}$, choosing $w$ appropriately in either case. We are concerned with the lower bound, and thus appeal to the following proposition, which follows from a trick of Rankin.

Proposition 2 ([10, Theorem 1, p. 52]). Suppose there is a constant $B>1$ so that

$$
\frac{1}{\log (z)} \sum_{p<z} \frac{\kappa(p) \log (p)}{p}<B
$$

for some $z \geq 2$. Writing $z=x^{1 / s}$, if $s>2 B$ we have

$$
G(\sqrt{x}) \geq \frac{1-e^{-C_{2}(s)}}{V\left(P\left(x^{1 / s}\right)\right)}
$$

where $C_{2}(s)=\frac{s}{2} \log (s / 2 B)-\frac{s}{2}+B$.

So we first find an upper-bound of the form

$$
\frac{1}{\log (z)} \sum_{p<z} \frac{\kappa(p) \log (p)}{p}<B
$$

An effective but simplistic upper-bound is easy to achieve. It is well known (e.g., see [28, Equation 3.24]) that $\frac{1}{\log (z)} \sum_{p<z} \frac{\log (p)}{p}<1$. Since $\kappa(p) \leq p_{0} \leq P$ we can take $B=P$, and then take $s=2 P+1$.

Next, we need to find an upper bound for $V(P(w))$, where $w=x^{1 / s}$. We begin by noting

$$
\begin{aligned}
V(P(w)) \leq \prod_{p<w}\left(1-\frac{1}{p}\right)^{\kappa(p)} & =\prod_{p<w}\left(1-\frac{1}{p}\right) \prod_{p<w, p \equiv 1} \prod_{(\bmod P)}\left(1-\frac{1}{p}\right)^{p_{0}-1} \\
& =\prod_{p<w}\left(1-\frac{1}{p}\right)^{p_{0}} \prod_{p<w, p \neq 1(\bmod P)}\left(1-\frac{1}{p}\right)^{-p_{0}+1} .
\end{aligned}
$$

Thus, it suffices to find an effective upper-bound for the Mertens' product over the arithmetic progression $1(\bmod P)$; or lower-bounds for the products over the other arithmetic progressions modulo $P$. The bounds given in [1] suffice. (To apply the results of that paper we may need to increase $P$ so we have $P \geq 37$, which can be 
done by adding primes to $\mathcal{P}$ if necessary.) We arrive at an effective inequality of the form

$$
V(P(w))<\frac{C_{3}}{\log (w)^{1+\frac{p_{0}-1}{\varphi(P)}}}
$$

where $C_{3}>0$ depends only on $P$, and $\varphi$ is the totient function.

At this point we still need to deal with the possibility that there might be prime factors of $N$ which are $1(\bmod P)$. Let $\mathcal{T}$ denote the set of all such prime factors, if any. We redefine $\Omega_{p}$ and $\kappa$, so that $\Omega_{p}=\{0(\bmod P)\}$ and $\kappa(p)=1$ whenever $p \in \mathcal{T}$. Notice that we now truly do have the bound

$$
\left|\left\{q \in Q_{p_{0}}: q<x\right\}\right| \leq|\mathcal{S}|+u .
$$

One of the effects of this change in the definition of $\Omega_{p}$ and $\kappa$ is that the needed constant $B$ is no larger, so we use the same constant $B=P$ as in our previous computation. The upper-bound on $V(P(w))$ is changed by a constant which depends only on the set $\mathcal{T}$. Further, the worst case is when $\mathcal{T}$ consists of the first $C_{1}$ primes which are $1(\bmod P)$. Letting $\mathcal{T}^{\prime}$ consist of the first $C_{1}$ primes which are $1(\bmod P)$, we have

$$
V(P(w))<\frac{C_{3}^{\prime}}{\log (w)^{1+\frac{p_{0}}{\varphi(P)}}}
$$

where

$$
C_{3}^{\prime}=C_{3} \prod_{p \in \mathcal{T}^{\prime}} \frac{1-\frac{1}{p}}{1-\frac{p_{0}}{p}}>0
$$

is still effectively computable. Note that at the cost of weaker bounds, we then have

$$
\left|\left\{q \in Q_{p_{0}}: q<x\right\}\right|<C_{4} x / \log (x)^{1+1 / \varphi(P)}
$$

where $C_{4}>0$ depends on $\mathcal{P}$, but is independent of $p_{0}$.

\section{Partial summation bound}

We are now ready to estimate $\prod_{q \in Q_{p_{0}}} \frac{q}{q-1}$. Suppose that all the prime factors of $N$ are greater than some integer $y>1$. By partial summation we have

$$
\begin{aligned}
\log & \left(\prod_{q \in Q_{p_{0}}} \frac{q}{q-1}\right)=-\sum_{q \in Q_{p_{0}}} \log (1-1 / q)=\sum_{q \in Q_{p_{0}}}\left(\frac{1}{q}+\frac{1}{2 q^{2}}+\frac{1}{3 q^{3}}+\cdots\right) \\
& <\int_{y}^{\infty} \frac{C_{4}}{x \log (x)^{1+1 / \varphi(P)}} d x+\sum_{q>y} \frac{1}{q(q-1)}<\frac{\varphi(P) C_{4}}{\log (y)^{1 / \varphi(P)}}+\sum_{n>y} \frac{1}{n(n-1)} \\
& =\frac{\varphi(P) C_{4}}{\log (y)^{1 / \varphi(P)}}+\frac{1}{y} .
\end{aligned}
$$

We can incorporate $1 / y$ into the main term (as the main term dominates), by changing $C_{4}$ to a new constant $C_{5}>0$. Thus,

$$
0<\log \left(C_{0}^{\prime}\right) \leq \log \left(\prod_{p_{0} \in \mathcal{P}} \prod_{q \in Q_{p_{0}}} \frac{q}{q-1}\right)<\frac{|\mathcal{P}| \varphi(P) C_{5}}{\log (y)^{1 / \varphi(P)}} .
$$


Solving for $y$ we have

$$
y<\exp \left(\left(\frac{|\mathcal{P}| \varphi(P) C_{5}}{\log \left(C_{0}^{\prime}\right)}\right)^{\varphi(P)}\right)
$$

and our third assumption on $C$ is that it is larger than the quantity on the right.

We have thus proven:

Theorem 3. Let $N>1$ be an odd integer with $\sigma_{-1}(N)=n / d$. Let $s \geq 1$ be a fixed integer, and let $\mathcal{P}$ be a finite set of primes. Suppose the prime factorization of $N$ has the form $N=\prod_{i=1}^{s} r_{i}^{a_{i}} \prod_{j=1}^{t} q_{j}^{b_{j}}$, where for each $j$ there is a prime $p_{j} \in \mathcal{P}$ with $p_{j} \mid\left(b_{j}+1\right)$. There exists a computable constant $C$, depending only on $\mathcal{P}, n$, and $s$, which gives an upper bound on the smallest prime factor of $N$.

\section{Our special Case, Dealing With SMall PRimes $p \neq 5$}

In the remainder of the paper we specialize to the case when $\sigma_{-1}(N)=2$, $\mathcal{P}=\{3,5\}$, and $s=1$. We further assume that the one prime factor of $N$ not necessarily limited by $\mathcal{P}$ is the special prime, $\pi$. Note that while the exponent of $\pi$ is not limited by $\mathcal{P}$ we do know that the exponent is $\equiv 1(\bmod 4)$. In particular, $\Phi_{2}(\pi)=\pi+1$ divides $2 N$. To abbreviate these assumptions, we refer to an integer $N$ satisfying the above conditions by the acronym R-OPN (a restricted odd perfect number).

Let $N$ be an R-OPN. Suppose for a moment that $Q_{5}$ is empty, and so all of the exponents of the non-special primes are $\equiv 2(\bmod 3)$. This implies that $\Phi_{3}(q) \mid N$ for all $q \mid N, q \neq \pi$. We wish to show that $3 \nmid N$ when $N$ is an R-OPN, working by contradiction. The next paragraph demonstrates how this is accomplished in one special case.

If $3 \mid N$, then since $\pi \neq 3$ we have $13=\Phi_{3}(3) \mid N$. In other words, starting with the prime 3 we are forced to have 13 as another prime divisor of $N$. We say that 3 contributes the prime factor 13 . Since $13 \equiv 1(\bmod 4)$ it might be the special prime, and so we will first consider that case. With $\pi=13$ we have $2 \cdot 7=\pi+1=\Phi_{2}(\pi) \mid 2 N$. This implies that $7 \in Q_{3}$ (since 13 is already the special prime, and we are assuming $\left.Q_{5}=\emptyset\right)$ and so 7 contributes the factors $3 \cdot 19=\Phi_{3}(7)$ to $N$. Repeating this argument we have that 19 contributes $3 \cdot 127=\Phi_{3}(19)$, which in turn contributes the factors $3 \cdot 5419=\Phi_{3}(127) \mid N$, and finally we have $3 \cdot 31 \cdot 313 \cdot 1009=\Phi_{3}(5419)$. We could continue this process of obtaining more and more factors of $N$, but pausing for a moment to collect our data we currently have $M=3^{4} \cdot 7^{2} \cdot 13^{1} \cdot 19^{2} \cdot 31^{2} \cdot 127^{2} \cdot 313^{2} \cdot 1009^{2} \cdot 5419^{2} \mid N$. On the other hand, $\sigma_{-1}(M)>2$. Adding more prime factors to $M$, or increasing the exponents on the factors we already have, only increases the size of $\sigma_{-1}(M)$, which contradicts $\sigma_{-1}(N)=2$.

One calls an integer $M>1$ for which $\sigma_{-1}(M)>2$ an abundant number. If $M \mid N$ and $M$ is abundant, then $N$ is abundant. Using this fact we were able to show that the situation in the previous paragraph led to a contradiction. However, there are more cases to consider; such as when 13 is not the special prime. The print-out below covers all cases. Some explanation of the notation in the print-out may be necessary. Every line is of the form " $p^{a} \Rightarrow \Phi_{a+1}(p)$ ", where $\Phi_{a+1}(p)$ is completely 
factored to tell us which new primes are contributed. The exponent $a$ is determined by the case under consideration (we have $a=1$ if $p$ is the special prime, and $a=2$ otherwise). Also note that the letter A tells us that the case under consideration gives us an abundant number, which allows us to back-track to the next case. The indentation tells us how far into a chain of factors we are, and also aids us in finding the next case to consider.

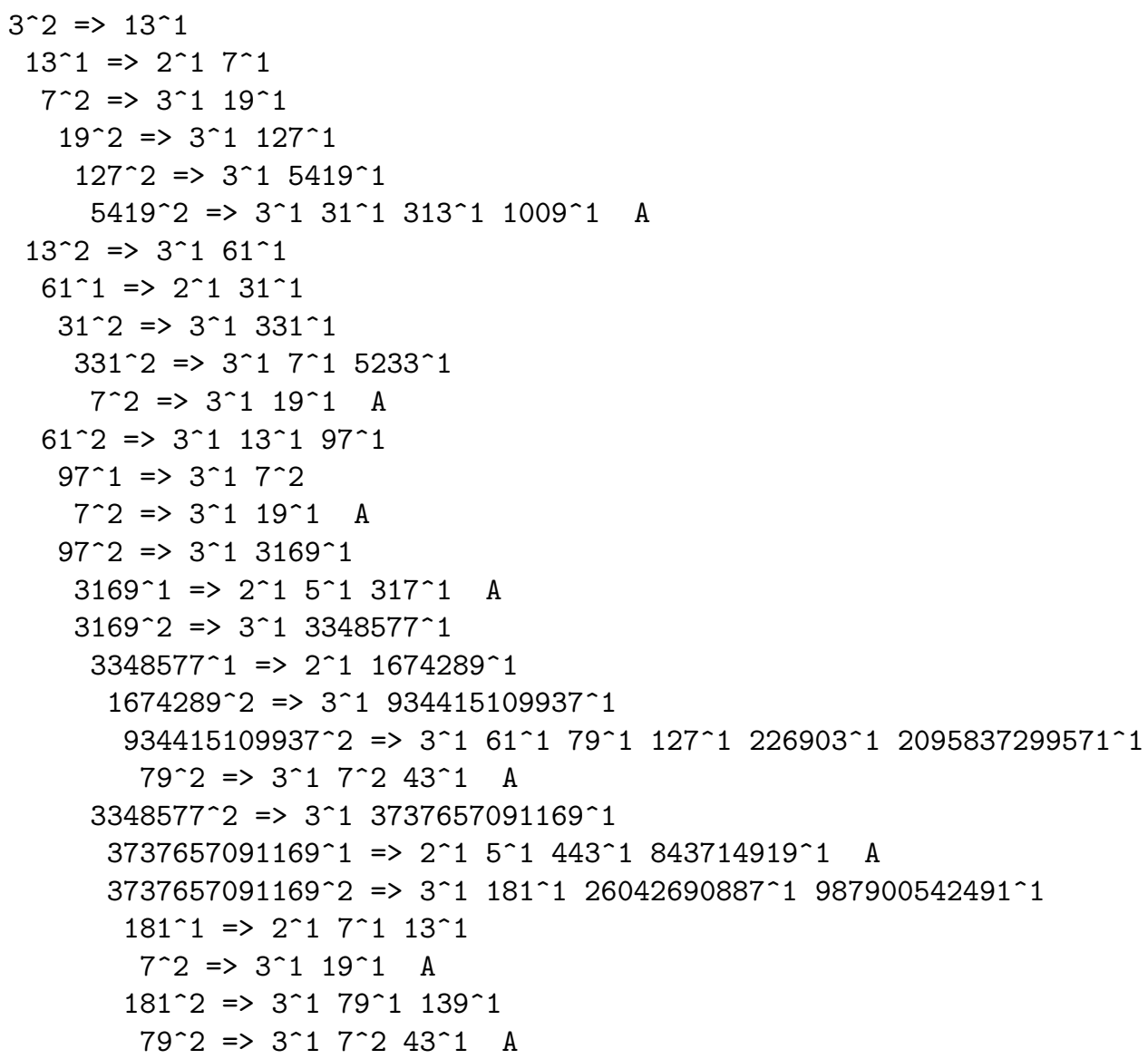

Note that the line 331^2 $=>3^{\wedge} 17^{\wedge} 1$ 5233^1 contributes the two primes 7 and 5233 , either of which we could use to start the next line. We chose 7 simply because it was the smallest prime we hadn't used, and we make similar choices throughout the chain. This algorithm above is often referred to as "creating a factor chain." Each possible chain of factors leads to a contradiction, i.e., the number must always become abundant. We thus can conclude $3 \nmid N$.

At this point we might ask what happens if we disregard our assumption that $Q_{5}$ is empty. This allows one more branch for each prime factor since we do not know whether we have $\Phi_{3}(q) \mid N$ or $\Phi_{5}(q) \mid N$. For example, instead of just dealing with 13 and $13^{2}$, we also have chains involving $13^{4}$. Surprisingly, the algorithm still successfully finishes in just under 1000 steps. In particular, if $N$ is an R-OPN, then $3 \nmid N$. 
We can try to repeat this process for the prime 5 , but the number of factors needed to achieve abundance increases dramatically. Even if we assume $Q_{3}$ is empty the number of cases to check is large; the chain must go to depths of about 80,000 prime factors which is entirely unfeasible. We describe how to overcome these difficulties in the next section. On the other hand, for any prime larger than 5 we quickly get a smaller prime (at least in practice) and thus reduce to a previous case. For example, the following print-out shows that if $7 \mid N$, then a smaller odd prime must divide $N$. When a smaller odd prime appears, we write S:

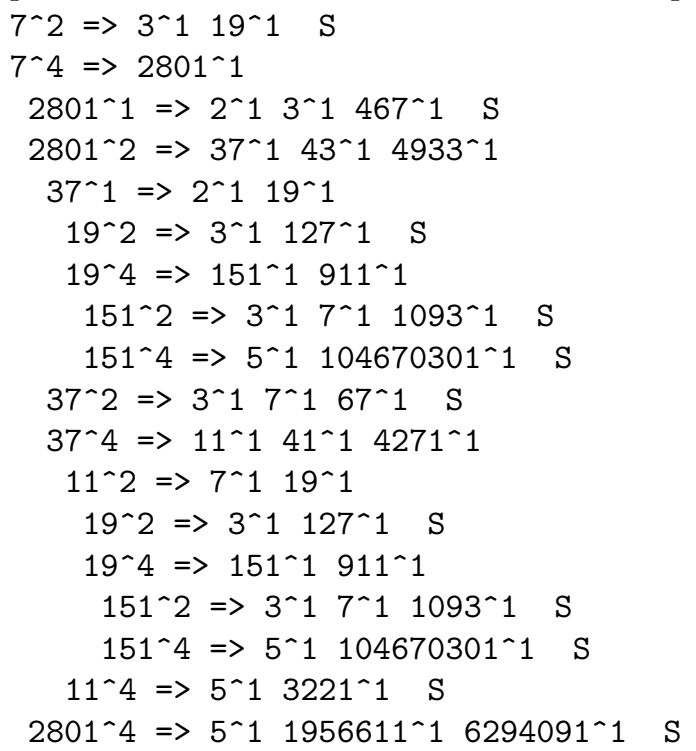

Notice that we do not even need to involve abundance computations. Continuing in this manner, one can prove that if $7 \leq q<10^{8}$ is prime and $q \mid N$, then a smaller odd prime divides $N$. Full print-outs (of these results, and the results of the next section) are available on the second author's website. With more effort, the upper bound on $q$ can be improved slightly; but a large improvement is not possible without improved factorization techniques (which is, of course, a very difficult problem!). Eventually the numbers we consider are too large to factor in a reasonable amount of time. It is interesting to note that $\Phi_{3}\left(\Phi_{5}(x)\right)=\left(x^{2}-x+1\right)\left(x^{6}+3 x^{5}+5 x^{4}+6 x^{3}+7 x^{2}+6 x+3\right)$, which helps factor some of the larger integers.

Computations suggest that any factor chain as above starting with a large prime quickly yields a smaller prime. It is an open question whether one can create a chain (using odd primes) which eventually yields no new prime factors.

\section{Dealing With the PRime 5}

We still need to deal with the case $q=5$, and then we can conclude that an R-OPN $N$ has no prime factors $<10^{8}$. It should be noted that even if we assume that $N$ is not divisible by a sixth power the computation is difficult because chains do not necessarily repeat prime factors. So we will continue to work with the assumption that $N$ is an R-OPN, even though our main interest lies in that special 
case. Keep in mind that we do know that $3 \nmid N$ from our work in the previous section.

The main difficulty in producing factor chains starting with the prime 5 is that abundance is more difficult to achieve. When we started with the prime 3 it fortunately contributed more to the abundance computations which helped in ending the chains quickly. To reach abundance when starting with 5 some cases will require hundreds of thousands of different primes. It should also be mentioned that moving from the case $\mathcal{P}=\{3\}$ to the case $\mathcal{P}=\{3,5\}$ increases the number of chains exponentially.

The key insight is to notice that certain primes occur quickly on any chain one considers. For example, we can always eventually get the prime 11 because of the following factor chain:

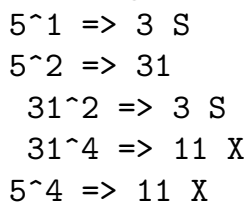

Note: To save room we do not print the full factorization of $\Phi_{a}(p)$ to the right of the arrow, which in some cases is difficult (or even impossible) to provide since full factorization is time intensive. Instead, we merely print the single prime factor we will use to continue the factor chain. So, for example, the last line tells us that $11 \mid \Phi_{5}(5)$ and that is all we need to know. Since we know 3 cannot divide $N$, hereafter we will drop all lines involving a 3 .

Now that we know that the prime 11 must occur on any chain we can use it to get other primes. A factor chain starting with the prime 11 which is seventy-two lines long tells us that 31 must occur in any chain. The prime 31 quickly gives us the prime 17351 , and in turn we get 41 using the chain:

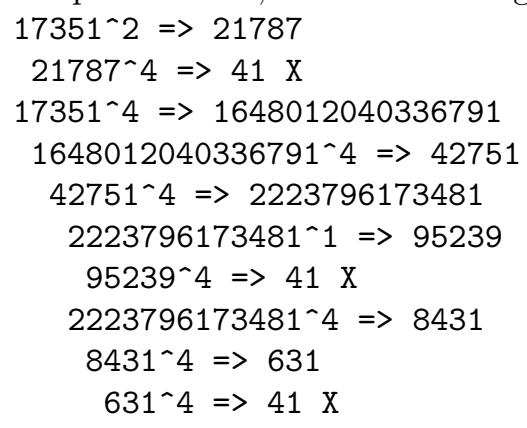

Note that we do not use the contradiction $\mathrm{S}$ when we have a prime smaller than 17351 (except on the dropped lines involving 3 ) since this chain is really a subchain of a factor chain starting with 5 .

In general, the only primes that we can capture in this way are all $\equiv 1(\bmod 5)$. This has to do with the fact that if $q \mid \Phi_{5}(p)$, then $q=5$ or $q \equiv 1(\bmod 5)$. This makes abundance computations more difficult (since we don't always have small primes like 7 and 13). However, it is possible to build a large list of primes that must occur in all chains. The ultimate goal is to collect enough primes, that each must occur in the chain, so that we reach abundance. 
Since we can ignore lines giving us 3 , and since $3 \mid \Phi_{3}(q)$ whenever $q \equiv 1(\bmod 3)$, one strategy that is highly effective is to branch along primes which are $1(\bmod 3)$ as much as possible. This allows us to avoid having to start lines with $q^{2}$. For example, once we have the prime 104670301 we get the prime 191 using the following factor chain:

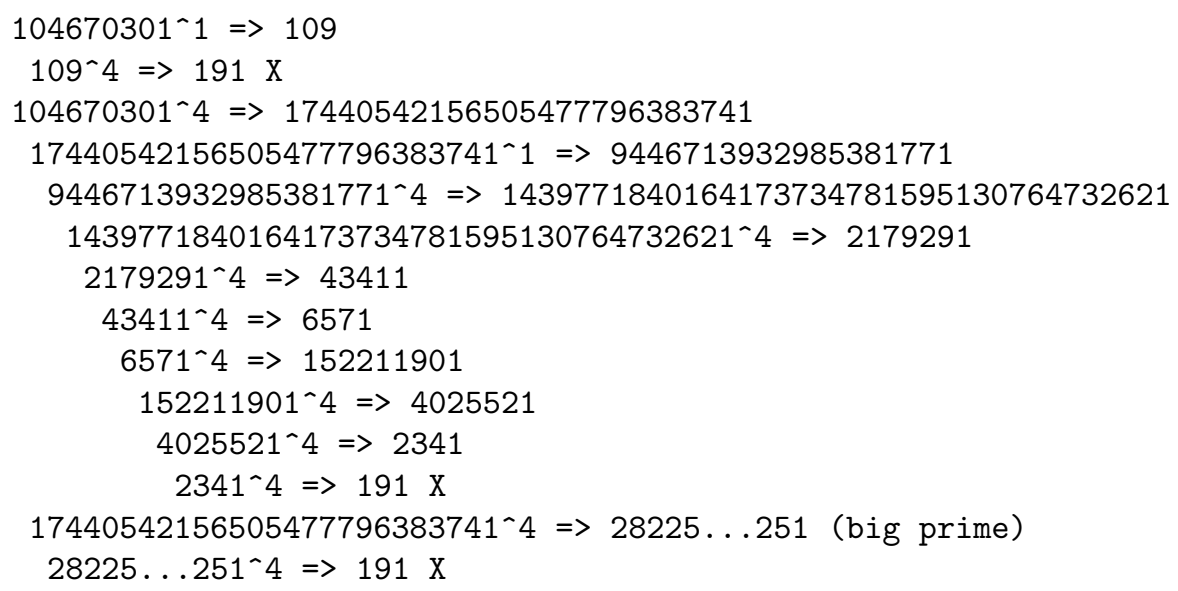

This strategy is extremely useful in the "endgame" when there are a lot of primes with which we can start chains.

The possibility that a prime can be the special prime has a quadratic effect on the number of lines in a given factor chain. But the special prime is special precisely because there is only one prime factor of $N$ with its properties. We can exploit this fact by creating two separate factor chains which involve distinct sets of possible special primes. If the special prime occurs in the first chain, then it cannot appear in the second chain and vice versa. Thus, we can write both chains without any contributions from a special prime, and at least one of them gives us the prime we need (since the special prime occurs in at most one of the two chains). To give an explicit example, suppose we want to get the prime 1051 and we already have the primes 241 and 104670301. Consider the two chains:

$241^{\wedge} 4 \Rightarrow 11106421$

$11106421^{\wedge} 4 \Rightarrow 3263466811411$

$3263466811411^{\wedge} 4 \Rightarrow 8821$

$8821^{\wedge} 4 \Rightarrow 1051 \mathrm{X}$

and

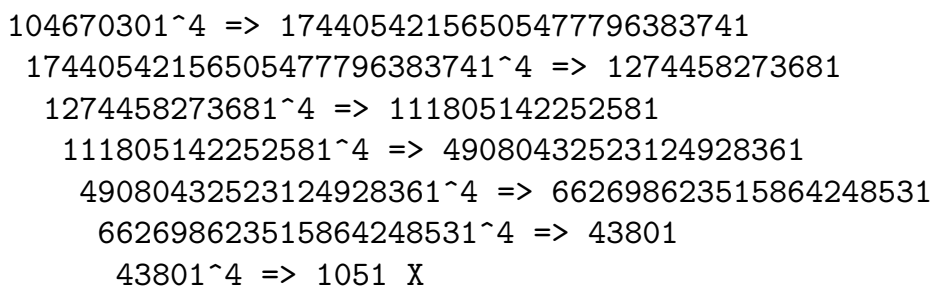

The only possible special primes which can appear in the first chain are 241,8821 
or 11106421 , since they are the only primes $p \equiv 1(\bmod 4)$. Thus, if the special prime is not one of these three primes, then the first chain shows us that we obtain 1051. On the other hand, if one of those three primes is the special prime, then the first chain is insufficient but the second chain suffices since it does not contain any of those possible special primes. This process reduces the quadratic effect of the special prime to a doubling effect.

There is one final simplifying technique that we put into use. Although it takes many primes to reach abundance, if a chain does ever involve the prime 7 (or other combinations of small primes) it is much easier to reach abundance. Thus, once we know that all chains eventually contain each of the first hundred primes congruent to $1(\bmod 5)$, we also know that if 7 occurs in a chain we have abundance. Hence, after that point we may ignore any line containing 7 . Similarly, with enough primes we can start ignoring lines with 13, 17, and so forth. Even before we know we can ignore 7 , we know that we can ignore chains which give us both 7 and 13 , or both 7 and 19. We put this into use when trying to obtain the prime 2531. We found two chains (without duplicated possible special primes) that either give us 2531 or 7 . We then found two new chains (again without duplicated possible special primes) that either give us 2531,13 , or 19 . Since 7 and 13 cannot happen together, and neither can 7 and 19 , we see that we must get 2531 .

After six months of running programs we wrote for Mathematica, we gathered enough chains and primes to achieve abundance. This proves that R-OPNs are not divisible by 5 .

\section{Minimizing the upper Bound}

Let $N$ be an R-OPN, and let $C$ be the upper bound we constructed for the smallest prime factor of $N$. Recall that our first assumption on $C$ was made to prevent $N$ from having prime factors in common with $n$, $d$, or $\mathcal{P}$. Due to the calculations above, we lose nothing in assuming $C>10^{8}$, and we achieve the same goal. It turns out this simple fact will greatly reduce a number of constants we deal with. For example, in the case we are considering we have $C_{0}=2$. Merely assuming that $C \geq \max \left\{P_{\max }, n, C_{0}\right\}+1=6$ we have $p_{1} \geq 6$ and so $C_{0}^{\prime} \geq 5 / 3$. But as we have proven that $C \geq 10^{8}$ we also have $C_{0}^{\prime} \geq 99999999 / 50000000=1.99999998$ which is a much better (indeed, nearly optimal) constant.

In all computations hereafter we will implicitly use the fact that $N$ has no prime factors less than $10^{8}$, and we will take $C_{0}^{\prime}=2\left(1-10^{-8}\right)$.

\section{WORKING WITH $Q_{3}$}

8.1. Defining $\Omega_{p}$ and $\kappa$. Our next job is to estimate $\prod_{q \in Q_{3}} \frac{q}{q-1}$. As before, we will use the large sieve. However, it turns out that we are able to improve our sieving sets $\Omega_{p}$ if we take into account the computations of the previous section. Notice that with $n=2$ we have $v_{3}(n)=v_{5}(n)=0$, and hence $C_{1}=1$. Thus, the only possible prime factor of $N$ congruent to $1(\bmod 15)$ is $\pi$, and we know $\pi>10^{8}$.

Let's recall some standard notation. For a prime $p$, and an integer $a \in \mathbb{Z}$ with $p \nmid a$, we let $o_{p}(a)$ denote the order of $a$ modulo $p$. In other words, this is the smallest positive integer for which $a^{o_{p}(a)} \equiv 1(\bmod p)$. If $r$ is a prime number and $r \mid(p-1)$, then there are exactly $r-1$ congruence classes mod $p$ which have order $r$. 
The abundance computations from the previous section were only useful for small primes. The reasons are two-fold. Primarily, when working with large primes we can end a chain much sooner by finding a smaller prime (which we will call the smaller prime contradiction). Secondarily, since we are assuming the prime factors of $N$ are larger than $10^{8}$ we would need an enormous number of primes to reach abundance. Because of these two reasons we wish to encode into the definition of the sets $\Omega_{p}$ as much information as we can glean from the smaller prime contradiction, and we can ignore abundance computations.

Fix $q \in Q_{3}$. We then know $\Phi_{3}(q) \mid N$. The simplest possible chain would occur if $\Phi_{3}(q)$ is divisible by a prime $p<10^{8}$. Such a prime is necessarily congruent to $1(\bmod 3)$, or equal to 3 , due to congruence restrictions on the possible prime factors of $\Phi_{3}(x)$; see [24, Lemma 1]. Thus, for all primes $p<10^{8}$ with $p \equiv 1(\bmod 3)$, we can sieve the two classes $a(\bmod p)$ with $o_{p}(a)=3$; and we can also sieve by the class $1(\bmod 3)$. This is a finite number of congruence classes, and we will see that it only changes the bound given in the large sieve by a constant factor.

The next simplest chain occurs as follows: Suppose we have a prime $p \mid \Phi_{3}(q)$. We may as well assume $q \equiv 2(\bmod 3)$ and so $p \equiv 1(\bmod 3)$. We then have $3 \mid \Phi_{3}(p)$, and hence $p \notin Q_{3}$. Therefore, either $p \in Q_{5}$ or $p=\pi$, and we can deal with the latter contingency as we did in previous sections. In the case $p \in Q_{5}$ the chain stops as long as $\Phi_{5}(p)$ is divisible by a prime $r<10^{8}$. By congruence conditions, we either have $r=5$ or $r \equiv 1(\bmod 5)$. In that case either $p \equiv 1(\bmod 15)$, or $o_{r}(p)=5$ and $p$ belongs to one of the four congruence classes modulo $r$ of order 5 . Unlike the previous case, this gives us an infinite set of congruence classes to sieve away. If one has good estimates for Mertens' products over arithmetic progressions, it would be valuable to not only sieve by primes in the class $1(\bmod 15)$ but in classes such as $1(\bmod 3) \cap a(\bmod 11)$ where $a=3,4,5,9$. The best explicit bounds we are aware of, that work for moduli that get increasingly large, are found in [1]. Unfortunately, even in the case of reasonable sized moduli, such as $k=165$, the bounds are too large to be of value in the computations we will perform.

We could continue this process, defining $\Omega_{p}$ to deal with chains of deeper depths. Complications arise in this situation. For example, we still have to deal with the possibility that one of the factors in the chain is the special prime. In practice we will restrict ourselves to chains of depth one, or of depth two if they yield 3 or 5 . Further, note that since the upper bound we reach for the smallest prime is $10^{1000}$ we may as well replace all instances of $10^{8}$ by the upper bound we wish to obtain. Due to technical conditions, we will work up to $10^{100}$.

We are now ready to define $\Omega_{p}$. Let $\mathcal{U}$ be the set of primes $p \equiv 1(\bmod 3)$ which are either $<10^{100}$ or $\equiv 1(\bmod 15)$. As just mentioned, depending on what tools are available, another choice of $\mathcal{U}$ may be appropriate. For an arbitrary prime $p$, set

$$
\Omega_{p}=\{0(\bmod p)\} \cup \begin{cases}\{1(\bmod 3)\} & \text { if } p=3, \\ \left\{a(\bmod p): o_{p}(a)=3\right\} & \text { if } p \in \mathcal{U}, \\ \emptyset & \text { otherwise. }\end{cases}
$$


Define

$$
\kappa(p)= \begin{cases}2 & \text { if } p=3 \\ 3 & \text { if } p \in \mathcal{U} \\ 1 & \text { otherwise }\end{cases}
$$

We have $\kappa(p)=\left|\Omega_{p}\right|$ just as before.

8.2. Finding bounds on $|\theta(x ; 15,1)-x / 8|$. In the following we let $\theta(t ; k, \ell)=$ $\sum_{p \leq t, p \equiv \ell(\bmod k)} \log (p)$. We first need to find explicit bounds on the error term for $\theta(x ; 15,1)-x / 8$. We begin with the following result found in [27, for values of $x$ that are small:

Lemma 4. For $1 \leq x \leq 10^{10}$ we have $|\theta(x ; 15,1)-x / 8| \leq 1.097307 \sqrt{x}$.

For slightly larger $x$ we follow [27, Section 4]. In the notation of that paper, taking $R=6.41$ and $C_{1}(\chi)=1$ due to work in [17] (which is also found in [19], and slightly improved in the preprint [18]), we get bounds of the form

$$
|\theta(x ; 15,1)-x / 8|<\epsilon x \text { for all } x>x_{0},
$$

where $\epsilon$ depends on $x_{0}$. We note that, without verifying the Riemann Hypothesis to greater heights for the Dirichlet L-function modulo 15, these bounds are only valid when $x_{0} \leq 10^{470}$. Here is a table of some of these values:

\begin{tabular}{cc}
$x_{0}$ & $\epsilon$ \\
\hline $10^{10}$ & 0.00903538 \\
$10^{11}$ & 0.00811594 \\
$10^{12}$ & 0.00780339 \\
$10^{20}$ & 0.00741842 \\
$10^{50}$ & 0.00646773 \\
$10^{100}$ & 0.00496541 \\
$10^{200}$ & 0.00241077 \\
$10^{400}$ & 0.00030846
\end{tabular}

For large values of $x$, we turn to the methods employed in [8]. In the notation of that paper, we take $R=9.645908801, H=2500$, and $C_{1}(\chi)=9.14$. In [8, Theorem 5], we have $X_{4}=10$ and so the bounds we obtain are accurate only when $\sqrt{\log (x) / R} \geq X_{4}$, or in other words, $x \geq 8.251 \cdot 10^{418}$. Putting it all together, we have

$$
|\theta(x ; 15,1)-x / 8|<0.77101532 \frac{x \log (x)^{1 / 4}}{e^{0.321979 \sqrt{\log (x)}}}
$$

when $x>10^{420}$.

8.3. Bounding a Mertens type product. We seek an effective bound on $\sum_{p \leq x, p \equiv 1(\bmod 15)} \frac{1}{p}$. By methods employed in [28], specifically in the derivation of equation (4.15) using partial summation (see also [4, Lemma 1.3.1]), we have

$$
\begin{aligned}
\sum_{\substack{p \leq x \\
p \equiv 1(\bmod 15)}} \frac{1}{p}= & \frac{1}{8} \log (\log (x))+M(15,1)+\frac{\theta(x ; 15,1)-x / 8}{x \log (x)} \\
& -\int_{x}^{\infty}(\theta(t ; 15,1)-t / 8) \frac{1+\log (t)}{t^{2} \log (t)^{2}} d t
\end{aligned}
$$


where $M(15,1)=-0.1506 \ldots$ is an explicit constant (see [21]). The work in the previous section yields $|\theta(x ; 15,1)-x / 8|<1.32812 x / \log (x)$ for all $x \geq 2$. Plugging this into the formula above, we then have

$$
\left|\sum_{\substack{p \leq x \\ p \equiv 1(\bmod 15)}} \frac{1}{p}-\frac{1}{8} \log (\log (x))-M(15,1)\right|<\frac{2}{\log (x)^{2}}+\frac{1.32812}{\log (x)} .
$$

As it will become useful shortly, we develop a series of inequalities. We have

$$
\frac{2}{\log (x)^{2}}+\frac{1.32812}{\log (x)}<\frac{1.48}{\log (x)} \text { when } x \geq 10^{6} .
$$

We also have

$$
\exp (-1.48 / \log (x))>1-\frac{1.48}{\log (x)}
$$

for $x \geq 2$, by a simple application of Taylor series remainders. Similarly,

$$
\exp (1.48 / \log (x))<1+\frac{1.65}{\log (x)}
$$

for $x \geq 10^{6}$. Next, we compute that

$$
\begin{aligned}
\sum_{\substack{p>x, n \geq 2 \\
p \equiv 1(\bmod 15)}} \frac{1}{n p^{n}} & <\frac{1}{2} \sum_{\substack{p>x, n \geq 2 \\
p \equiv 1(\bmod 15)}} \frac{1}{p^{n}}=\frac{1}{2} \sum_{\substack{p>x \\
p \equiv 1(\bmod 15)}} \frac{1}{p(p-1)} \\
& <\frac{1}{2} \sum_{n>x} \frac{1}{n(n-1)}<\frac{1}{2(x-1)}
\end{aligned}
$$

and so,

$$
\exp \left(\sum_{\substack{p>x, n \geq 2 \\ p \equiv 1(\bmod 15)}} \frac{1}{n p^{n}}\right)<\exp (1 / 2(x-1))<1+\frac{1.1}{x}=\alpha(x)
$$

for $x \geq 10^{6}$. Finally,

when $x \geq 10^{6}$.

$$
\alpha(x)\left(1+\frac{1.65}{\log (x)}\right)<1+\frac{1.66}{\log (x)}
$$

Taking the logarithm of a product, expanding the Taylor series, and taking exponentials we obtain

$$
\prod_{\substack{p \leq x \\ p \equiv 1(\bmod 15)}}\left(1-\frac{1}{p}\right)=\exp \left[-\sum_{\substack{p \leq x \\ p \equiv 1(\bmod 15)}} \frac{1}{p}-\sum_{\substack{n \geq 2 \\ p \equiv 1(\bmod 15)}} \frac{1}{n p^{n}}+\sum_{\substack{p>x, n \geq 2 \\ p \equiv 1(\bmod 15)}} \frac{1}{n p^{n}}\right] .
$$

Using the bounds given above, we then have, for $x \geq 10^{6}$,

$$
\frac{C(15,1)}{\log (x)^{1 / 8}}\left(1-\frac{1.48}{\log (x)}\right)<\prod_{\substack{p \leq x \\ p \equiv 1(\bmod 15)}}\left(1-\frac{1}{p}\right)<\frac{C(15,1)}{\log (x)^{1 / 8}}\left(1+\frac{1.66}{\log (x)}\right)
$$

where $C(15,1)=1.1617 \ldots$ according to 22 . 
8.4. Bounding another Mertens type product. Again, using the methods employed in [28, we have

$$
\begin{aligned}
\sum_{\substack{p \leq x \\
p \equiv 1(\bmod 3)}} \frac{1}{p}= & \frac{1}{2} \log (\log (x))+M(3,1)+\frac{\theta(x ; 3,1)-x / 2}{x \log (x)} \\
& -\int_{x}^{\infty}(\theta(t ; 3,1)-t / 2) \frac{1+\log (t)}{t^{2} \log (t)^{2}} d t
\end{aligned}
$$

where $M(3,1)=-0.35689 \ldots$ is an explicit constant (again see 21]). By 8], we have the bound $|\theta(t ; 3,1)-t / 2|<0.262 \frac{t}{\log (t)}$ for $t \geq 1531$. Repeat the process in the last subsection to obtain

$$
\left|\sum_{\substack{p \leq x \\ p \equiv 1(\bmod 3)}} \frac{1}{p}-\frac{1}{2} \log (\log (x))-M(3,1)\right|<\frac{0.393}{\log (x)^{2}}+\frac{0.262}{\log (x)} .
$$

For $x \geq 10^{6}$ we have

$$
\frac{0.393}{\log (x)^{2}}+\frac{0.262}{\log (x)}<\frac{0.3}{\log (x)}
$$

Taylor series remainders prove that

and we also have

$$
\exp (-0.3 / \log (x))>1-\frac{0.3}{\log (x)}
$$

Thus, for $x \geq 10^{6}$ we have

$$
\alpha(x) \exp (0.3 / \log (x))<1+\frac{0.31}{\log (x)} .
$$

$$
\frac{C(3,1)}{\log (x)^{1 / 2}}\left(1-\frac{0.3}{\log (x)}\right)<\prod_{\substack{p \leq x \\ p \equiv 1(\bmod 3)}}\left(1-\frac{1}{p}\right)<\frac{C(3,1)}{\log (x)^{1 / 2}}\left(1+\frac{0.31}{\log (x)}\right)
$$

where $C(3,1)=1.403477 \ldots$ according to 22 .

8.5. Estimating $B$. Now, consider the quantity

$$
\frac{1}{\log (z)} \sum_{p<z} \frac{\kappa(p) \log (p)}{p} \leq \frac{1}{\log (z)}\left(\sum_{p<z} \frac{\log (p)}{p}+\frac{\log (3)}{3}+\sum_{\substack{p<z \\ p \equiv 1(\bmod 3)}} \frac{2 \log (p)}{p}\right) .
$$

A simple computation shows that the quantity on the right is smaller than 2 for $z \leq 10^{6}$. In the calculations below, we implicitly assume $z>10^{6}$ unless otherwise stated.

We first estimate the piece involving the arithmetic progression. Recall that by $\left[8\right.$, we have the bound $|\theta(t ; 3,1)-t / 2|<0.262 \frac{t}{\log (t)}$ for $t \geq 1531$. By partial summation, we compute for integer values of $z$ that

$$
\begin{aligned}
\sum_{p \leq z, p \equiv 1(\bmod 3)} \frac{\log (p)}{p} & =\theta(z ; 3,1) \frac{1}{z}+\int_{t=7}^{z} \theta(t ; 3,1) \frac{1}{t^{2}} d t \\
& <\frac{1}{2}+0.262 \frac{1}{\log (z)}+\frac{1}{2} \log (z)+0.262 \log (\log (z))-1.14
\end{aligned}
$$


which is increasing in $z$. In particular,

$$
\frac{1}{\log (z)} \sum_{p \leq z, p \equiv 1(\bmod 3)} \frac{2 \log (p)}{p} \leq 1+0.524 \frac{\log (\log (z))}{\log (z)}-\frac{1.2}{\log (z)}
$$

for all real $z>10^{6}$. The function $0.524 \frac{\log (\log (z))}{\log (z)}-\frac{1.2}{\log (z)}$ is bounded above by 0.02 .

By equation (3.22) in [28, we have

$$
\sum_{p \leq z} \frac{\log (p)}{p}<\log (z)+E+\frac{1}{2 \log (z)}
$$

for $z \geq 319$, where $E=-1.33258 \ldots$ is a constant. Putting all of this together, we have

$$
\frac{1}{\log (z)} \sum_{p<z} \frac{\kappa(p) \log (p)}{p}<2.02
$$

for all $z \geq 2$. Hence, we take $B=2.02$. Notice that this choice of $B$ continues to work with any of the other choices for $\mathcal{U}$ discussed above. Asymptotically, $B=2$ would be the optimal constant when $\mathcal{U}$ consists of all primes $p \equiv 1(\bmod 3)$.

8.6. Estimating $V(P(w))$. Another result of Dusart [7] tells us that $\frac{e^{-\gamma}}{\log (w)}\left(1-\frac{0.2}{\log (w)^{2}}\right)<\prod_{p<w}\left(1-\frac{1}{p}\right)<\frac{e^{-\gamma}}{\log (w)}\left(1+\frac{0.2}{\log (w)^{2}}\right), \quad$ for $w>2973$.

Rewriting $V(P(w))$, for $w \geq 10^{100}$ we have

$$
\begin{aligned}
V(P(w))= & \frac{1}{2} \prod_{p \leq w}\left(1-\frac{1}{p}\right) \prod_{\substack{p \leq 10^{100} \\
p \equiv 1(\bmod 3)}}\left(1-\frac{1}{p}\right)^{2} \\
& * \prod_{\substack{p \leq w \\
p \equiv 1(\bmod 15)}}\left(1-\frac{1}{p}\right)^{2} \prod_{\substack{p \leq 10^{100} \\
p \equiv 1(\bmod 15)}}\left(1-\frac{1}{p}\right)^{-2} \\
& * \prod_{\substack{p \leq 10^{100} \\
p \equiv 1(\bmod 3)}} \frac{\left(1-\frac{3}{p}\right)}{\left(1-\frac{1}{p}\right)^{3}} \prod_{\substack{p \leq w \\
p \equiv 1}} \frac{\left(1-\frac{3}{p}\right)}{\left(1-\frac{1}{p}\right)^{3}} \prod_{\substack{p \leq 10^{100} \\
p \equiv 1(\bmod 15)}} \frac{\left(1-\frac{1}{p}\right)^{3}}{\left(1-\frac{3}{p}\right)} .
\end{aligned}
$$

The first two products in the third line are both bounded above by 1 . The third product on the third line can be bounded by

$$
\begin{aligned}
& \prod_{\substack{p \leq 10^{100} \\
p \equiv 1(\bmod 15)}} \frac{\left(1-\frac{1}{p}\right)^{3}}{\left(1-\frac{3}{p}\right)}=\prod_{\substack{p \leq 10^{6} \\
p \equiv 1(\bmod 15)}} \frac{\left(1-\frac{1}{p}\right)^{3}}{\left(1-\frac{3}{p}\right)} \prod_{\substack{10^{6}<p \leq 10^{100} \\
p \equiv 1(\bmod 15)}} \frac{\left(1-\frac{1}{p}\right)^{3}}{\left(1-\frac{3}{p}\right)} \\
&<\prod_{\substack{p \leq 10^{6} \\
p \equiv 1(\bmod 15)}} \frac{\left(1-\frac{1}{p}\right)^{3}}{\left(1-\frac{3}{p}\right)} \prod_{10^{6}<n \leq 10^{100}} \frac{\left(1-\frac{1}{n}\right)^{3}}{\left(1-\frac{3}{n}\right)}<1.00482 .
\end{aligned}
$$


The other products were bounded previously, so we have for $w \geq 10^{100}$ that

$$
\begin{aligned}
V(P(w))< & \frac{1}{2}(1.00482) \frac{e^{-\gamma}}{\log (w)}\left(1+\frac{0.2}{\log \left(10^{100}\right)^{2}}\right) \frac{C(3,1)^{2}}{\log \left(10^{100}\right)}\left(1+\frac{0.31}{\log \left(10^{100}\right)}\right)^{2} \\
& * \frac{C(15,1)^{2}}{\log (w)^{1 / 4}}\left(1+\frac{1.66}{\log \left(10^{100}\right)}\right)^{2} \frac{\log \left(10^{100}\right)^{1 / 4}}{C(15,1)^{2}}\left(1-\frac{1.48}{\log \left(10^{100}\right)}\right)^{-2} \\
< & \frac{0.00969}{\log (w)^{5 / 4}} .
\end{aligned}
$$

Recall that we must deal with the possibility of a single element $\pi \in \mathcal{T}$, which is accomplished by multiplying our answer above by $\left(1-\frac{1}{10^{8}}\right) /\left(1-\frac{3}{10^{8}}\right)$, as $\pi>10^{8}$. This quantity changes our constants so little that with some forethought it can be accounted for in previous computations.

8.7. Picking a value for $s>2 B$. We set $w=x^{1 / s}$, and assume $w>10^{100}$. By Proposition 2 and the work in the previous section, we have

$\frac{1}{G(\sqrt{x})} \leq\left(\left(1-e^{-C_{2}(s)}\right)^{-1} V\left(P\left(x^{1 / s}\right)\right)\right)<0.00969\left(1-e^{-C_{2}(s)}\right)^{-1} \log (x)^{-5 / 4} s^{5 / 4}$

with $C_{2}(s)=\frac{s}{2} \log (s / 2 B)-\frac{s}{2}+B$ and $B=2.02$. This quantity is minimized when $s$ is a little smaller than 10 . We take $s=10$ and obtain

$$
\frac{1}{G(\sqrt{x})} \leq \frac{0.219}{\log (x)^{5 / 4}}
$$

Notice that since $w>10^{100}$ we are also assuming $x>10^{1000}$.

8.8. Measuring the size of $Q_{3}$. In the large sieve, taking $u=\sqrt{x}$ where $x>$ $10^{1000}$, we have

$$
\left|\left\{q \in Q_{3}: q<x\right\}\right| \leq \frac{2 x}{G(\sqrt{x})}+\sqrt{x}<\frac{0.438 x}{\log (x)^{5 / 4}} .
$$

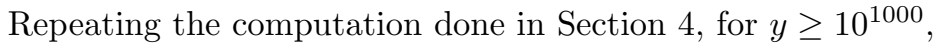

$$
\begin{aligned}
& \log \left(\prod_{q \in Q_{3}} \frac{q}{q-1}\right)=-\sum_{q \in Q_{3}} \log (1-1 / q)=\sum_{q \in Q_{3}}\left(\frac{1}{q}+\frac{1}{2 q^{2}}+\frac{1}{3 q^{3}}+\cdots\right) \\
& <\int_{y}^{\infty} \frac{0.438}{x \log (x)^{5 / 4}} d x+\sum_{q>y} \frac{1}{q(q-1)}<\frac{1.752}{\log (y)^{1 / 4}}+\sum_{n>y} \frac{1}{n(n-1)}<\frac{1.76}{\log (y)^{1 / 4}} .
\end{aligned}
$$

\section{WORKING WITH $Q_{5}$}

We now turn our attention to the set $Q_{5}$. As many of the same computations and remarks that were made with regards to $Q_{3}$ hold for the new set, we will simply work quickly through the appropriate bounds. We will often continue our assumption that $w>10^{100}$ (unless otherwise posited). 
9.1. Defining $\Omega_{p}$ and $\kappa$. We take $\mathcal{U}$ to be the set of primes $q \equiv 1(\bmod 5)$ which also either satisfy $q<10^{100}$ or $q \equiv 1(\bmod 15)$. Set

$$
\Omega_{p}=\{0(\bmod p)\} \cup \begin{cases}\{1(\bmod 5)\} & \text { if } p=5, \\ \left\{a(\bmod p): o_{p}(a)=5\right\} & \text { if } p \in \mathcal{U}, \\ \emptyset & \text { otherwise }\end{cases}
$$

and define

$$
\kappa(p)= \begin{cases}2 & \text { if } p=5 \\ 5 & \text { if } p \in \mathcal{U} \\ 1 & \text { otherwise }\end{cases}
$$

9.2. Estimating $B$. Computations suggest (just as before) that $B=2$ is the optimal value, asymptotically. We will get close to this value. We begin with the following bounds found in 27.

Proposition 5. For $t>10^{10}$,

$$
\left|\theta(t ; 5,1)-\frac{t}{4}\right|<0.002785 \frac{t}{4} .
$$

For all $0<t \leq 10^{10}$,

$$
\left|\theta(t ; 5,1)-\frac{t}{4}\right|<1.412480 \sqrt{t}
$$

For integer values of $z>10^{10}$, we then have

$$
\begin{aligned}
\sum_{p \leq z, p \equiv 1(\bmod 5)} \frac{\log (p)}{p} & =\theta(z ; 5,1) \frac{1}{z}+\int_{t=11}^{z} \theta(t ; 5,1) \frac{1}{t^{2}} d t \\
& <0.2507 \log (z)+0.5 .
\end{aligned}
$$

Combining this with work done in previous sections, we have

$\frac{1}{\log (z)} \sum_{p<z} \frac{\kappa(p) \log (p)}{p} \leq \frac{1}{\log (z)}\left(\sum_{p<z} \frac{\log (p)}{p}+\frac{\log (5)}{5}+\sum_{\substack{p<z \\ p \equiv 1(\bmod 5)}} \frac{4 \log (p)}{p}\right)<2.05$

for all real $z>10^{10}$.

For integer values in the range $10^{5}<z<10^{10}$, we have

$$
\begin{aligned}
\sum_{p \leq z, p \equiv 1(\bmod 5)} \frac{\log (p)}{p} & =\theta(z ; 5,1) \frac{1}{z}+\int_{t=11}^{z} \theta(t ; 5,1) \frac{1}{t^{2}} d t \\
& <0.25 \log (z)+0.503-1.41 / \sqrt{z}
\end{aligned}
$$

In particular, $\frac{1}{\log (z)} \sum_{p<z} \frac{\kappa(p) \log (p)}{p} \leq 2.09$ for all real $z>10^{5}$. Finally, for $z<10^{5}$ one checks directly that 2 works as an upper bound. We may then take $B=2.09$. 
9.3. Finding bounds on $|\theta(x ; 5,1)-x / 4|$. We bound $|\theta(x ; 5,1)-x / 4|$ using the techniques of Section 8.2 By Proposition 5 , for $1 \leq x \leq 10^{10}$ we have $\mid \theta(x ; 15,1)-$ $x / 8 \mid \leq 1.097307 \sqrt{x}$. For slightly larger $x$ we again follow [27, Section 4]. For very large $x$ we use 8 . Putting these computations together we have the bound

$$
|\theta(x ; 15,1)-x / 8|<0.6 \frac{x}{\log (x)}
$$

for all $x \geq 10$.

\subsection{Bounding a third Mertens type product. We have the equality}

$$
\begin{aligned}
\sum_{\substack{p \leq x \\
p \equiv 1(\bmod 5)}} \frac{1}{p}= & \frac{1}{4} \log (\log (x))+M(5,1)+\frac{\theta(x ; 5,1)-x / 4}{x \log (x)} \\
& -\int_{x}^{\infty}(\theta(t ; 5,1)-t / 4) \frac{1+\log (t)}{t^{2} \log (t)^{2}} d t
\end{aligned}
$$

where $M(5,1)=-0.2088344 \ldots$ is a constant. Using the bound given in the last subsection, we have for $x \geq 10^{10}$ that

$$
\left|\sum_{\substack{p \leq x \\ p \equiv 1(\bmod 5)}} \frac{1}{p}-\frac{1}{4} \log (\log (x))-M(5,1)\right|<\frac{0.9}{\log (x)^{2}}+\frac{0.6}{\log (x)} .
$$

For $x \geq 10^{6}$ we have

$$
\frac{0.9}{\log (x)^{2}}+\frac{0.6}{\log (x)}<\frac{0.67}{\log (x)}
$$

Letting $x \geq 10^{6}$, Taylor remainders yield

$$
\exp (0.67 / \log (x))<1+\frac{0.71}{\log (x)},
$$

and since by the previous computation we have

$$
\exp \left(\sum_{\substack{p>x, n \geq 2 \\ p \equiv 1(\bmod 5)}} \frac{1}{n p^{n}}\right)<\exp (1 / 2(x-1))<1+\frac{1.1}{x}=\alpha(x)
$$

we also have

As

$$
\alpha(x)\left(1+\frac{0.71}{\log (x)}\right)<1+\frac{0.72}{\log (x)}
$$

$$
\prod_{\substack{p \leq x \\ p \equiv 1(\bmod 5)}}\left(1-\frac{1}{p}\right)=\exp \left[-\sum_{\substack{p \leq x \\ p \equiv 1(\bmod 5)}} \frac{1}{p}-\sum_{\substack{n \geq 2 \\ p \equiv 1(\bmod 5)}} \frac{1}{n p^{n}}+\sum_{\substack{p>x, n \geq 2 \\ p \equiv 1(\bmod 5)}} \frac{1}{n p^{n}}\right],
$$

we obtain

$$
\prod_{\substack{p \leq x \\ p \equiv 1(\bmod 5)}}\left(1-\frac{1}{p}\right)<\frac{C(5,1)}{\log (x)^{1 / 4}}\left(1+\frac{0.72}{\log (x)}\right)
$$

where $C(5,1)=1.2252 \ldots$ according to $[22$. 
9.5. Estimating $V(P(w))$. We have for $w>10^{100}$,

$$
\begin{aligned}
V(P(w))< & \frac{3}{4}(1.00482) \prod_{p \leq w}\left(1-\frac{1}{p}\right) \prod_{\substack{p \leq 10^{100} \\
p \equiv 1(\bmod 5)}}\left(1-\frac{1}{p}\right)^{4} \\
& * \prod_{\substack{p \leq w \\
p \equiv 1(\bmod 15)}}\left(1-\frac{1}{p}\right)^{4} \prod_{\substack{p \leq 10^{100} \\
p \equiv 1(\bmod 15)}}\left(1-\frac{1}{p}\right)^{-4} \\
< & \frac{0.0673}{\log (w)^{3 / 2}} .
\end{aligned}
$$

9.6. Picking a value for $s>2 B$. We take $s=10>2 B$, and so $x>10^{1000}$ as before. We obtain

$$
\frac{1}{G(\sqrt{x})} \leq \frac{2.78}{\log (x)^{3 / 2}}
$$

9.7. Measuring the size of $Q_{5}$. In the large sieve, taking $u=\sqrt{x}$ where $x>$ $10^{1000}$, we have

$$
\left|\left\{q \in Q_{5}: q<x\right\}\right| \leq \frac{2 x}{G(\sqrt{x})}+\sqrt{x}<\frac{5.56 x}{\log (x)^{3 / 2}} .
$$

Repeating the computation done in Section 4, for $y \geq 10^{1000}$,

$$
\begin{aligned}
& \log \left(\prod_{q \in Q_{5}} \frac{q}{q-1}\right)=-\sum_{q \in Q_{5}} \log (1-1 / q)=\sum_{q \in Q_{5}}\left(\frac{1}{q}+\frac{1}{2 q^{2}}+\frac{1}{3 q^{3}}+\cdots\right) \\
& <\int_{y}^{\infty} \frac{5.56}{x \log (x)^{3 / 2}} d x+\sum_{q>y} \frac{1}{q(q-1)}<\frac{11.12}{\log (y)^{1 / 2}}+\sum_{n>y} \frac{1}{n(n-1)}<\frac{11.2}{\log (y)^{1 / 2}}
\end{aligned}
$$

\section{Putting it all together}

Recall that $C_{0}^{\prime}=2\left(1-10^{-8}\right)$. Our computations tell us that

$$
\log \left(C_{0}^{\prime}\right)>\frac{1.76}{\log (y)^{1 / 4}}+\frac{11.2}{\log (y)^{1 / 2}}
$$

for $y \geq 10^{1000}$. Thus, $y$ is larger than the smallest prime divisor of our purported number, and this finishes the proof of the Main Theorem. This improves Yamada's result by seven orders of magnitude.

\section{IDEALIZATION}

One might wonder what bounds are possible if we idealize the situation slightly. First, we may as well assume $C_{0}=2$. Second, we will suppose that $\pi$ has no further effect on the computations. Third, let's take $\mathcal{U}$ to be the set of all primes $p \equiv 1(\bmod 3)$ when working with $Q_{3}$, and take $\mathcal{U}$ to be the set of all primes $p \equiv 1(\bmod 5)$ when working with $Q_{5}$. While Rankin's trick gives us effective bounds, it is not tight. By methods in [16, Chapter 1] and [10, Theorem 2.2.2], 
one can find an asymptotic and effective bound on $G(w)$. In fact, for $Q_{3}$ we have $G(w) \sim c_{3, g} \log (w)^{2}$ where

$$
c_{3, g}=\frac{e^{-2 \gamma}}{\Gamma(1+2)} \lim _{w \rightarrow \infty} \frac{1}{\log (w)^{2} V(P(w))}=0.164 \ldots
$$

Similarly, for $Q_{5}$ we have $G(w) \sim c_{5, g} \log (w)^{2}$ where

$$
c_{5, g}=\frac{e^{-2 \gamma}}{\Gamma(1+2)} \lim _{w \rightarrow \infty} \frac{1}{\log (w)^{2} V(P(w))}=0.145 \ldots
$$

Fourth, let's suppose we are in a sieving situation where $|\mathcal{S}| \leq x / G(\sqrt{x})$. Ignoring error terms we have

$$
\log \left(\prod_{q \in Q_{3}} \frac{q}{q-1}\right) \approx \sum_{q \in Q_{3}} \frac{1}{q} \approx \int_{y}^{\infty} \frac{6.1}{x \log (x)^{2}} d x \approx \frac{6.1}{\log (y)}
$$

and similarly,

$$
\log \left(\prod_{q \in Q_{5}} \frac{q}{q-1}\right) \approx \frac{6.9}{\log (y)} .
$$

Solving the inequality

$$
\log (2) \leq \frac{13}{\log (y)}
$$

yields $y \lesssim 1.4 \cdot 10^{8}$.

With a significant amount of work we could improve the lower bound in the Main Theorem to $10^{10}$, or possibly to $10^{12}$ if the computation was distributed. This would easily surpass the idealized bound we obtained above. However, that bound is still not obtainable for two reasons. First, the choices for the sets $\mathcal{U}$ are not realistic. Second, and more importantly, obtaining effective asymptotic bounds for $G(w)$ with current techniques seems to require $w \gg 10^{10}$, and hence we are led to something close to $x \gg 10^{20}$, which is just too large.

\section{Open questions AND FUtURE DIRECTIONS}

Let $p$ be a prime and let $q \mid \Phi_{3}(p)$ be a prime divisor. Either $q=3$ or $\Phi_{3}(q)$ is divisible by 3 . Similarly, if $q \mid \Phi_{5}(p)$ is prime, then either $q=5$ or $\Phi_{5}(q)$ is divisible by 5 . Thus, if we consider factor chains where new primes arise only from either factoring $\Phi_{3}(p)$ or $\Phi_{5}(p)$, and we can stop whenever 3 or 5 occurs, then the factor chain simply bounces back and forth between applying $\Phi_{3}$ and $\Phi_{5}$. Eventually, we expect one of the prime factors to be $q \equiv 1(\bmod 15)$, and then both $\Phi_{3}(q)$ and $\Phi_{5}(q)$ terminate our factor chain.

In practice, such chains reach primes $q \equiv 1(\bmod 15)$ very quickly. It would be interesting to prove that for all sufficiently large primes $p$, factor chains limited as above always reach 3 or 5 . Moreover, it would be interesting to prove (or disprove) that there is a bound on the depth it takes to reach 3 or 5 .

One can also ask: What happens if we expand our set of limited exponents to $\mathcal{P}=\{3,5,7\}$ ? In this case, each prime in a factor chain has three possible branches (and possibly a fourth, if it is the special prime). Our technique for dealing with the prime 5 is inadequate because no specific primes appears (at least quickly) in every chain, although in principle one should still be able to deal with 3 because abundance computations are less difficult in that case. 


\section{ACKNOWLEDGEMENTS}

We wish to thank William Lipp and Carlos Pinho for their help factoring large integers. We also thank the referee for the many suggestions which improved our paper.

\section{REFERENCES}

1. Olivier Bordellès, An explicit Mertens' type inequality for arithmetic progressions, J. Inequal. Pure Appl. Math. 6 (2005), no. 3, Article 67, 10 pp. (electronic). MR2164308 (2006g:11192)

2. R. P. Brent, G. L. Cohen, and H. J. J. te Riele, Improved techniques for lower bounds for odd perfect numbers, Math. Comp. 57 (1991), no. 196, 857-868. MR.1094940 (92c:11004)

3. Graeme L. Cohen and Ronald M. Sorli, On the number of distinct prime factors of an odd perfect number, J. Discrete Algorithms 1 (2003), no. 1, 21-35, Combinatorial algorithms. MR 2016472 (2004h:11003)

4. Alina Carmen Cojocaru and M. Ram Murty, An introduction to sieve methods and their applications, London Mathematical Society Student Texts, vol. 66, Cambridge University Press, Cambridge, 2006. MR2200366 (2006k:11184)

5. R. J. Cook, Bounds for odd perfect numbers, Number theory (Ottawa, ON, 1996), CRM Proc. Lecture Notes, vol. 19, Amer. Math. Soc., Providence, RI, 1999, pp. 67-71. MR1684591 (2000d:11010)

6. Leonard Eugene Dickson, Finiteness of the odd perfect and primitive abundant numbers with $n$ distinct prime factors, Amer. J. Math. 35 (1913), no. 4, 413-422. MR.1506194

7. Pierre Dusart, Inégalités explicites pour $\psi(X), \theta(X), \pi(X)$ et les nombres premiers, $\mathrm{C} . \mathrm{R}$. Math. Acad. Sci. Soc. R. Can. 21 (1999), no. 2, 53-59. MR.1697455 (2000f:11125)

8. __ Estimates of $\theta(x ; k, l)$ for large values of $x$, Math. Comp. 71 (2002), no. 239, 11371168 (electronic). MR 1898748 (2003f:11139)

9. Takeshi Goto and Yasuo Ohno, Odd perfect numbers have a prime factor exceeding $10^{8}$, Math. Comp. 77 (2008), no. 263, 1859-1868. MR.2398799 (2009b:11008)

10. George Greaves, Sieves in number theory, Ergebnisse der Mathematik und ihrer Grenzgebiete (3) [Results in Mathematics and Related Areas (3)], vol. 43, Springer-Verlag, Berlin, 2001. MR.1836967 (2002i:11092)

11. Otto Grün, Über ungerade vollkommene Zahlen, Math. Z. 55 (1952), 353-354. MR0053123 $(14: 724 \mathrm{~g})$

12. Kevin G. Hare, New techniques for bounds on the total number of prime factors of an odd perfect number, Math. Comp. 76 (2007), no. 260, 2241-2248 (electronic). MR 2336293 (2008g:11006)

13. D. R. Heath-Brown, Odd perfect numbers, Math. Proc. Cambridge Philos. Soc. 115 (1994), no. 2, 191-196. MR1277055 (96b:11130)

14. Douglas E. Iannucci, The second largest prime divisor of an odd perfect number exceeds ten thousand, Math. Comp. 68 (1999), no. 228, 1749-1760. MR1651761(2000i:11200)

15. - The third largest prime divisor of an odd perfect number exceeds one hundred, Math. Comp. 69 (2000), no. 230, 867-879. MR.1651762(2000i:11201)

16. Henryk Iwaniec and Emmanuel Kowalski, Analytic number theory, American Mathematical Society Colloquium Publications, vol. 53, American Mathematical Society, Providence, RI, 2004. MR2061214 (2005h:11005)

17. Habiba Kadiri, An explicit zero-free region for Dirichlet L-functions, submitted, found at http://www.cs.uleth.ca/ kadiri/articles.

18. $\longrightarrow$ An explicit zero-free region for the Dirichlet L-functions, found at http://www.arxiv.org/abs/math/0510570.

19. _ـ Une région explicite sans zéro pour les fonctions $L$ de Dirichlet, Thèse, Université de Lille I, U.F.R. de Mathématiques - Laboratoire A.G.A.T.-U.M.R. 8524.

20. Masao Kishore, On odd perfect, quasiperfect, and odd almost perfect numbers, Math. Comp. 36 (1981), no. 154, 583-586. MR606516 (82h:10006)

21. A. Languasco and A. Zaccagnini, Computing the Mertens and Meissel-Mertens constants for sums over arithmetic progressions, Experiment. Math. 19 (2010), no. 3, 279-284. MR2743571 (2011j:112247) 
22. - On the constant in the Mertens product for arithmetic progressions. II. Numerical values, Math. Comp. 78 (2009), no. 265, 315-326. MR2448709 (2010g:11164)

23. Wayne L. McDaniel, The non-existence of odd perfect numbers of a certain form, Arch. Math. (Basel) 21 (1970), 52-53. MR0258723 (41:3369)

24. Pace P. Nielsen, Odd perfect numbers have at least nine distinct prime factors, Math. Comp. 76 (2007), no. 260, 2109-2126 (electronic). MR2336286 (2008g:11153)

25. Paul Pollack, On Dickson's theorem concerning odd perfect number, American Math. Monthly 118 (2011), no. 2, 161-164.

26. Carl Pomerance, Multiply perfect numbers, Mersenne primes, and effective computability, Math. Ann. 226 (1977), no. 3, 195-206. MR0439730 (55:12616)

27. Olivier Ramaré and Robert Rumely, Primes in arithmetic progressions, Math. Comp. 65 (1996), no. 213, 397-425. MR.1320898 (97a:11144)

28. J. Barkley Rosser and Lowell Schoenfeld, Approximate formulas for some functions of prime numbers, Illinois J. Math. 6 (1962), 64-94. MR0137689 (25:1139)

29. Tomohiro Yamada, On the divisibility of odd perfect numbers by the power of a prime, preprint, found at http://arxiv.org/abs/math/0511410, $13 \mathrm{pp}$.

30. _ Odd perfect numbers of a special form, Colloq. Math. 103 (2005), no. 2, 303-307. MR 2197857 (2006h:11007)

Department of Mathematics, Brigham Young University, Provo, Utah 84602

E-mail address: adam3.14159@gmail.com

Department of Mathematics, Brigham Young University, Provo, Utah 84602

E-mail address: pace@math.byu.edu

CNRS, Lab. J.V. Poncelet, Moscow LRI, Bât. 490, Univ. Paris-Sud 11, 91405, Orsay Cedex, France

E-mail address: ochem@lri.fr 